AROUEOLOGía Y SOCIEDAD

№ 26, 2013: 191-206

ISSN: $0254-8062$

RECIBIDO: 21 / MAR. / 2013

ACEPTADO: 18 / ABR. / 2013

\title{
LA INTERACCIÓN CULTURAL PREHISPÁNICA DE LOS VALLES INTERANDINOS, EL SUBANDINOY LA AMAZONIA, NORTE DE ECUADOR
}

\author{
JORGE ARELLANO \\ Departamento de ANTROPOlOgía, SMithsonian INSTITUTION \\ arellanoA@si.edu
}

\section{RESUMEN}

El presente artículo muestra la importancia de los eventos medioambientales para las culturas prehispánicas de las hoyas interandinas de Ecuador para la elección de las rutas de contacto con el subandino y la Amazonía. En este sentido, se analizan los datos arqueológicos registrados en una de las rutas de acceso al subandino utilizadas por las culturas de la hoya interandina de Quito-Guayllabamba, para conectarse con el piedemonte amazónico. Esta ruta estuvo controlada por la Fase de cerámica Cosanga, conocida históricamente como etnia Quijos. Las evidencias arqueológicas sugieren que esta cultura del subandino también pudo haber sido la intermediaria para contactos con culturas del bajo Amazonas. Por su parte los datos arqueológicos relacionados con la Tradición amazónica de cerámica corrugada-falsocorrugada muestran, que grupos de esta tradición trataron de expandirse hacia el subandino siguiendo el valle del río Quijos. Al parecer el avance de ambas culturas fue detenido por los eventos medioambientales producidos por la actividad volcánica de El Reventador.

Palabras Clave: Eventos medioambientales, contactos prehispánicos, Andes-Subandino y Amazonía, Norte de Ecuador.

\begin{abstract}
This article shows the importance of the environmental events for the pre-Hispanic cultures of the InterAndean basins of Ecuador to choose the right paths to make contact with Sub-Andean and Amazonian cultures. Here, we analyze archaeological data recorded in one of the paths used by the Quito-Guayllabamba valley cultures to the Sub-Andean hills and Amazonian foothills. This route was controlled by the ceramic Phase Cosanga, historically known as Quijos. Archaeological evidence suggests that this culture may have been the intermediary for contacts with the lower Amazon. Meanwhile archaeological data related to the Amazonian ceramic Tradition corrugated-falsocorrugated shows that this tradition tried to expand into the Sub-Andean hills following the Quijos River valley. The expansion of both cultures may have been stop by the constant environmental events product of the volcanic activity of El Reventador.
\end{abstract}

KeYwords: Environmental events, pre-Hispanic contacts, Andes-Subandean hills-Amazonia, Northern Ecuador. 


\section{INTRODUCCIÓN}

El subandino Norte y piedemonte amazónico al este de los Andes del Ecuador, tuvo en el pasado una importancia muy específica porque sirvió de catalizador entre las culturas andinas y las amazónicas. A través de la región geográfica comúnmente denominada subandino, «montaña» o alta Amazonía se realizaron los contactos inter-étnicos con los pueblos asentados en la baja Amazonía, en principio para intercambio de recursos naturales y posteriormente en la introducción de nuevas ideas de tipo religioso.

Las tierras altas de la cadena andina oriental desde Colombia por el norte hasta el nudo de Vilcanota por el sur en Perú, están conectadas a las tierras amazónicas por las nacientes de los principales ríos que pertenecen a la cuenca hidrográfica del río Amazonas. Estos canales naturales fueron tradicionalmente mencionados como las rutas de contacto entre las culturas andinas y amazónicas.

En Ecuador tres grandes sub-cuencas hidrográficas asociadas con la cuenca Amazónica fueron las rutas empleadas para los contactos entre culturas prehispánicas. En el Norte la sub-cuenca del río Napo, en el centro la del Pastaza y por el sur la de Morona-Santiago por medio del río Upano. Actualmente una pequeña sub-cuenca, la del río Aguarico, adquiere importancia por el registro de nuevas evidencias arqueológicas.

Sin lugar a dudas, la sub-cuenca del Napo fue una de las más importantes y de la que más noticias se tuvo desde los primeros años de la conquista española. Los ríos que provienen del norte de la cordillera andina tienen como colectores principales los ríos Aguarico, Quijos-Coca y Napo. El valle del Aguarico está relacionado con las hoyas o valles inter-andinos del Chota y Carchi a través de las nacientes del río Cofanes. Las escasas evidencias arqueológicas al este de las montañas sugieren que las culturas prehispánicas del subandino estuvieron en contacto con las sociedades del Chota y Carchi (Delgado 2009). En el borde oriental de estas dos hoyas se encuentran instalaciones de una serie de «pucaras» o fortalezas estratégicamente asociadas a los accesos naturales que tienen dirección al pie de monte y Amazonía alta (Plaza 1976, 1977). De manera similar se menciona que los sitios multi-étnicos de Pimampiro están asociados con la interacción entre sierra y oriente (Porras 1972; Bray 2005).

Por su parte el valle del Quijos tiene acceso a la hoya o valle inter-andino de GuayllabambaQuito por medio de las nacientes del río Papallacta en la cordillera de Yanaurco, a través del paso de Guamani a $4000 \mathrm{~m}$ de altura (Fig. 1). Los principales datos de la presencia de contactos entre sierra y subandino provienen de las investigaciones arqueológicas realizadas por Porras $(1975,1985)$ en gran parte del valle de Quijos y el río Cosanga, donde fue determinada la tradición cerámica denominada Cosanga. Este tipo de cerámica denominada Panzaleo en la Sierra ecuatoriana, también sugiere un contacto entre las hoyas interandinas del norte y el piedemonte al este de los Andes como producto de comercio para usos ceremoniales (Bray 1995).

La siguiente ruta de contacto se ubica en la región que se encuentra dominada por las nacientes del río Upano. En esta región los contactos prehispánicos estuvieron concentrados principalmente entre Sierra y culturas del subandino a través de los sitios de la Tradición Upano (Porras 1987; Salazar 1998, 2008; Rostain 1999).

Por último en el extremo sur del Ecuador, en los contrafuertes andinos de Tzunantza, el río Zamora-Santiago y las nacientes del río Numbatá fueron las tradicionales rutas de los pueblos asentados en la hoya del Catamayo para su contacto con el subandino y la Amazonía en dirección al Marañon (Ledergerber 2006; Guffroy 1984, 1987). De manera similar, las investigaciones de Ledergerber (2006) en la zona del piedemonte de Morona y Santiago sugieren que en esta zona los contactos de los pueblos entre sierra, subandino y Amazonía fueron más frecuentes y que a partir de esta se podrían haber desplazado hacia el norte del Perú.

Las anteriores rutas de contacto cruzan invariablemente las sierras montañosas en pasos accesibles abiertos durante la mayor parte del año. No obstante es posible que hayan existido algunos otros 


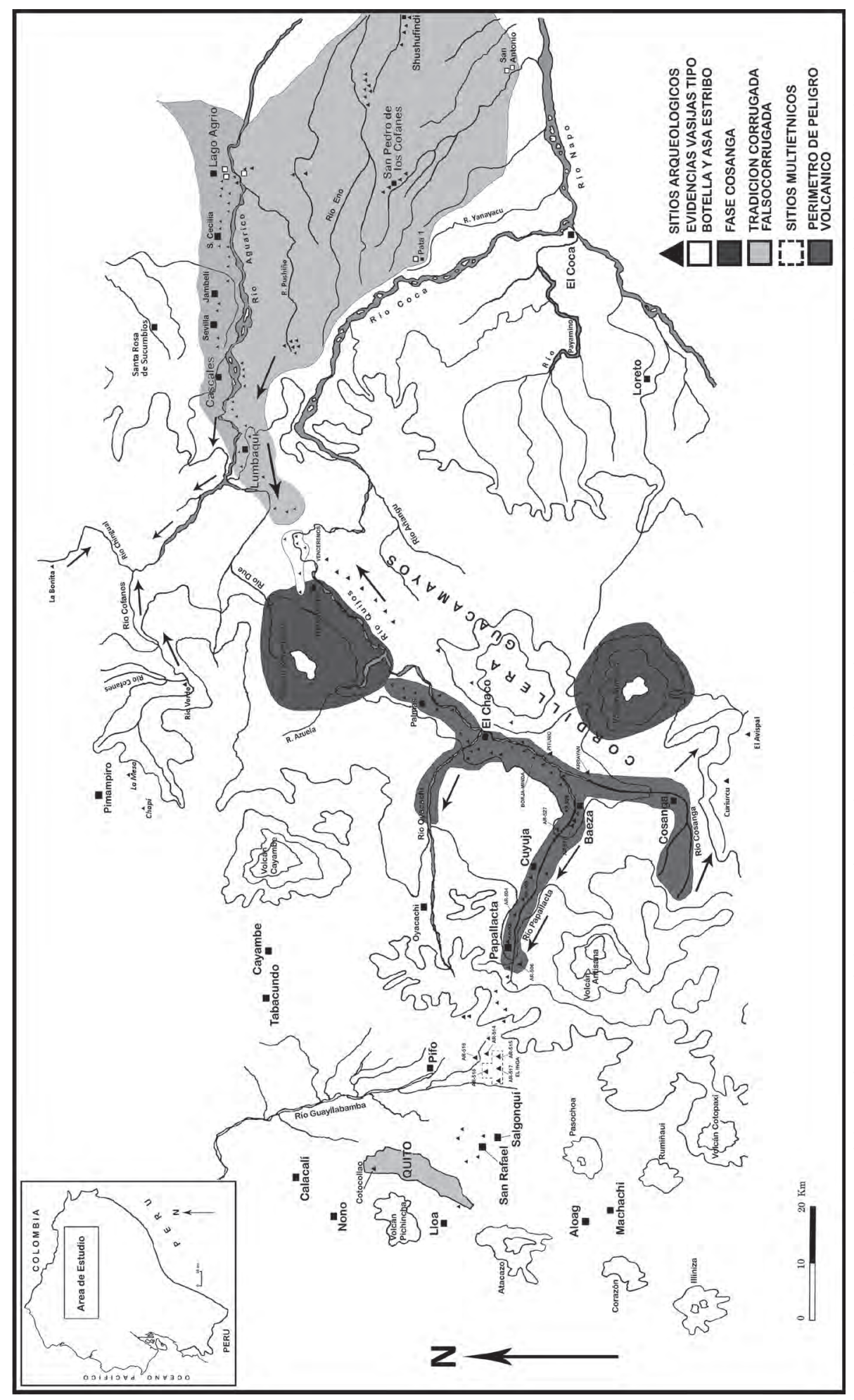


pasos en la región del valle de Cebadas (Arellano 1994,1997) con dirección a las serranías de Osogoche y Atillo cuyo borde oriental está asociado al volcán Sangay.

El presente estudio analiza los datos arqueológicos obtenidos durante la primera fase de la prospección arqueológica a lo largo de la línea propuesta y alternativas para la construcción del oleoducto transecuatoriano de crudos pesados (Arellano 1999; Salazar et al. 1999). Esta línea que tuvo un trazo siguiendo una de las principales rutas de acceso que tuvieron las culturas del subandino, proporcionó datos para considerar que culturas del piedemonte amazónico del norte también trataron de tener contacto con la Sierra central del Ecuador. Sin embargo los datos de eventos medioambientales sugieren que en varios períodos este contacto fue interrumpido y solo realizado de manera ocasional. Además, sugiere la posibilidad que tuvieron las culturas de la Amazonía baja para emplear a las culturas de pie de monte como intermediarios para una relación con la Sierra.

\section{LoS REgistros ARQUEólogicos DEL SUBANDINO A LA SiERRA}

Las investigaciones arqueológicas a lo largo de la línea del oleoducto transecuatoriano comprendieron varias fases, de las cuales la primera consistió en una prospección sistemática desde el puerto de Balao en la costa ecuatoriana hasta la ciudad amazónica de lago Agrio. Los datos arqueológicos obtenidos entre la población de San Rafael en la hoya de Guayllabamba-Quito y la localidad de Baeza en la ceja oriental de montaña provienen del registro de 23 sitios en los cuales el principal material cultural es cerámica fragmentada de la fase Cosanga (Salazar et al. 1999). Por su parte en las prospecciones en la cordillera de Guacamayos, entre las poblaciones de Lumbaqui y Linares, se ubicaron 27 sitios con evidencias de cerámica (Arellano 2000). De manera complementaria se realizó reconocimientos en las serranías marginales al este de la Hoya de Quito, Alto Yaruqui y Alto Pomasqui (2800 msnm). En estas serranías se ubicaron ocho sitios con evidencias de material cultural de diversas culturas y períodos (Arellano 2000b).

Estos sitios, incluidos a los mencionados por Porras (1975) en la determinación de la fase Cosanga y los registrados y estudiados por Cuellar $(2006,2010)$ y Yepez (2008), forman un conjunto de evidencias que señalan a la etnia que ocupó el valle del río Quijos como el principal intermediario en el pie de montaña para la relación amazónica con las etnias del valle central de Quito.

El análisis tipológico de la cerámica de sitios multi-componentes ubicados en la región de Pimampiro sugiere que si bien existió una relación con la fase Cosanga con intercambio de productos, está fue solo completamente establecida en el periodo de integración con la difusión de productos específicos como por ejemplo la chicha de mandioca y vasijas como objetos funerarios (Bray 1995: 152).

Por su parte los datos a lo largo del flanco occidental de la cordillera indican que existe una franja de sitios multi-componentes, los cuales fueron utilizados como mercados de transacción de productos. El material cerámico de estos sitios está conformado principalmente por un apreciable porcentaje del tipo engobado en rojo típico de la sierra central y emparentado con la fase ChilibuloChillogallo. Las formas reconstruidas incluyen cuencos y ollas globulares trípodes de patas cónicas. En estos contextos también se encuentran asociados fragmentos de la fase Cosanga (Panzaleo para otros) caracterizados por su delgado espesor y calidad de acabado con decoración de líneas paralelas de color rojo sobre una superficie marrón grisácea. La presencia de la cultura Inca está registrada por sus típicos fragmentos con motivos decorativos en negro sobre rojo y la colonia está representada por fragmentos con acabado superficial vidriado (Arellano 2000).

Siguiendo el curso del río Papallacta con dirección a sus nacientes se pudo comprobar que la parte del valle entre Papallacta y Baeza fue ocupado por la fase Cosanga desde periodos tempranos, ya que en algunos sitios se recuperó material cerámico de los tipos denominados Bermejo grueso y Pituro negro pulido, determinados por Cuellar (2010) en sitios del valle de Cosanga. Esta asociación de 
tipos Cosanga y Bermejo fueron registrados en tres de los diez sitios del valle del Papallacta. Entre estos el de Huila previamente estudiado por Porras (1975). Mientras que la asociación con el tipo Pituro se encuentra en un solo sitio, Maspa (AR-503), que se encuentra geográficamente localizado a los 2500 msnm en lo que vendría a constituir el límite superior de la ceja de montaña. Los sitios Jambihuasi (AR-537) y el Control (AR-536) tienen solo material Cosanga, mientras Burruyacu (AR-529) ubicado en la terrazas de Banco Samana (Porras, 1975) y Alejandría (AR-527), se presentan los tipos Bermejo grueso y Cosanga.

Entre los 2500 a 3500 msnm las ocupaciones pertenecen a la fase Cosanga ocasionalmente con presencia del tipo denominado Papallacta ordinario que esta caracterizado por una cerámica de 2 a $9 \mathrm{~mm}$ de espesor y acabado en brochado con el empleo de manojo de hojas (Porras 1975: 117). No obstante, según Cuellar (2010) estas características son similares a las del tipo Bermejo grueso proveniente de los sitios ubicados en Santa Lucia del Bermejo. El conjunto de sitios en Chalpi Chico (AR-504A, B y C) se encuentra integrado a terrazas actualmente utilizadas para el cultivo de papa. Los sitios son multi-componentes y contienen tres tipos de material cerámico entre los cuales el tipo Cosanga es predominante. El siguiente tipo corresponde al Papallacta ordinario y el tercero está caracterizado por un acabado bruñido en marrón rojizo. Este último no pertenece a las etnias del Alto o Bajo Amazonas y probablemente fue transportado por una corriente que viene de la sierra. Los sitios que se ubican en los límites inferiores del paramo, Papallacta (AR-505) y laguna Papallacta (AR-506) también contienen una variedad de tipos cerámicos, en especial el sitio AR-505 que presenta cuatro tipos al margen del tipo Cosanga que es el más importante por su porcentaje. El segundo tipo en importancia es el Papallacta Ordinario y luego le siguen Alisado, Engobado en Anaranjado y Marrón en Rojo Bruñido.

En el margen oeste de la divisoria de aguas de la cordillera, los sitios ubicados en el páramo del Tablón no presentan ningún material cultural cerámico, pero si contienen algunos artefactos de obsidiana y lascas. El primer sitio con evidencias cerámicas se encuentra a $3255 \mathrm{msnm}$, en el cerro Itulcachi (AR-513), en este se nota la integración de un tipo netamente indígena de paredes gruesas, superficie alisada con engobe marrón rojizo con un tipo colonial de cerámica vidriada en verde y amarillo. La única forma reconstruida del tipo indígena corresponde a una vasija de un apreciable tamaño elaborada en falso torno con borde ligeramente evertido, labio ensanchado. La unión del borde con el cuello de la vasija muestra huellas de dedos a pesar del alisado. En la colección de este tipo existen asas de corte elipsoidal por lo que se infiere que estas vasijas estaban provistas de las mismas.

A partir de este sitio, los demás se encuentran directamente relacionados con culturas o etnias indígenas que poblaron la hoya de Guayllabamba-Quito. Los que tienen relación con Cosanga son los ubicados en los márgenes de la cordillera como por ejemplo en el sector del Inga (AR-514, 515 y 516). Sin embargo, el material Cosanga es limitado y está conformado por restos de vasijas de la forma con pedestal y cuencos. El tipo cerámico dominante es el que presenta un engobe marrón rojizo sobre una superficie que puede ser alisada o pulida. Las formas comunes son vasijas globulares con borde evertido y vasijas de la variedad compoteras con bases anulares. En el sitio AR-516 al margen de estos tipos se tiene la presencia de cerámica Inca. (Fig. 2)

De manera similar los sitios Barrotieta (AR-517) y ciudadela La Vicuña (AR-518) tienen material cerámico que puede ser directamente integrado a los del Inga. Si bien la cerámica Cosanga está presente en cada sitio no representa un porcentaje de importancia. Las formas reconocidas son vasijas globulares pequeñas con el cuerpo decorado con diseños geométricos lineares, característica decorativa de Cosanga. El tipo más frecuente es engobado en marrón de varias tonalidades, variables entre el oscuro al rojizo anaranjado, de paredes gruesas ( 8 a $15 \mathrm{~mm}$ de espesor) y desgrasante o antiplástico de arena. Las formas reconstruídas corresponden a vasijas globulares con base plana y cuencos con base anular, no presentan ninguna decoración y sus restos se encuentran esparcidos en superficie, en gran parte de la hoya de Guayllabamba. 


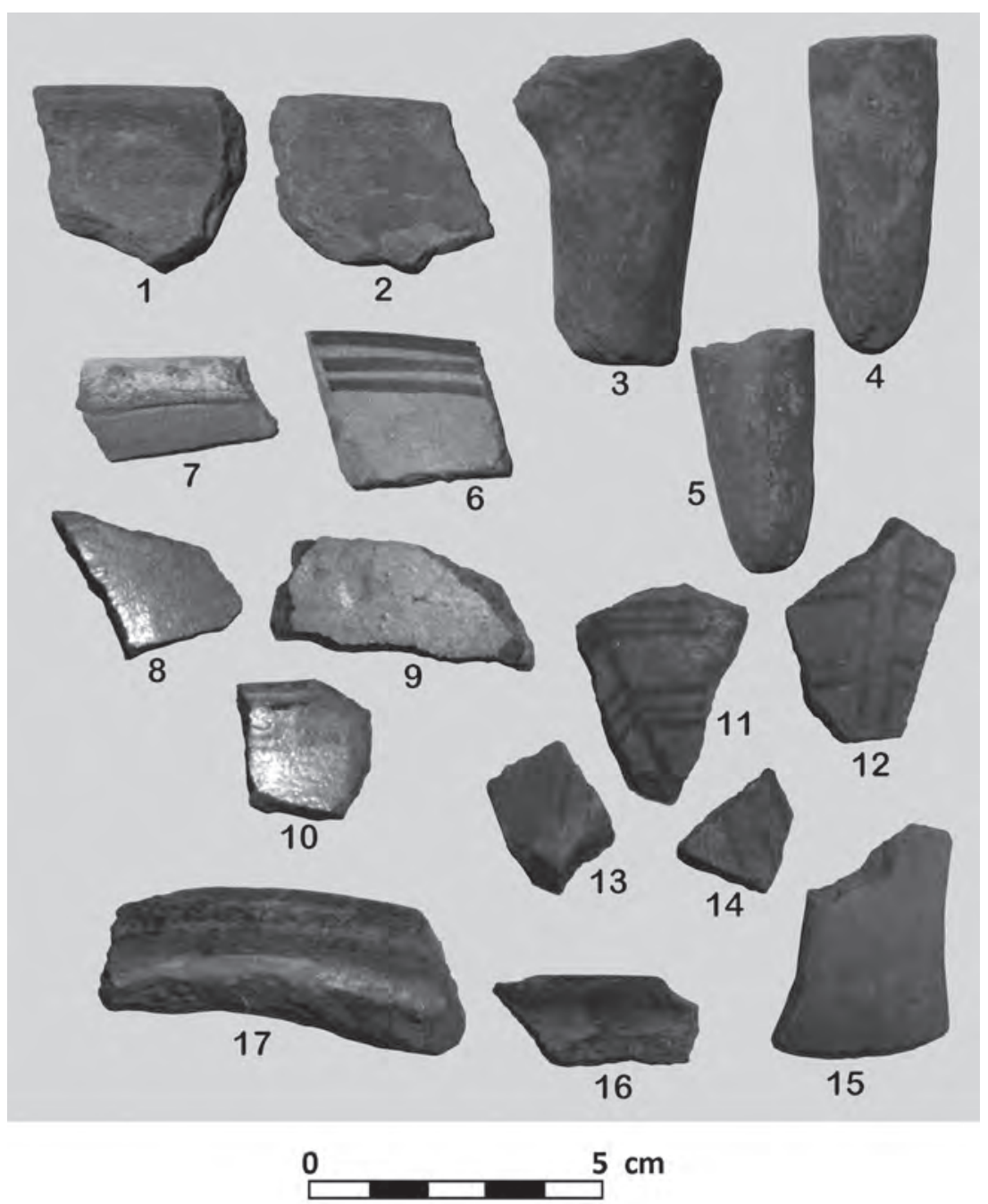

Figura 2. Fragmentos de cerámica provenientes de los sitios multiétnicos de El Inga. Hoya Interandina de Quito-Guayllabamba. 1-5 Fase Chilibulo-Chillogallo; 6 Fase Cosanga, 7-10 Colonial, 11-14 Inca Cañari, 15-17 Inca Caranqui (Fotografía del autor)

Los sitios registrados entre la ciudadela La Vicuña y la ciudad de Quito no contienen evidencias de la fase Cosanga y solo se presenta la variedad local descrita anteriormente que lamentablemente carece de estudios descriptivos y no tiene relación con una específica etnia del valle de Quito. El análisis más cercano a este tipo de cerámica de paredes gruesas y engobada en marrón rojizo, se encuentra en el estudio del material cultural de la Pucara de Rumicucho (Almeida y Jara 1984: 64).

Es interesante indicar que en la mayor parte de los sitios arqueológicos que se encuentran en el valle de Quito se ubican fragmentos de vasijas coloniales, los cuales pueden clasificarse tipológicamente por su acabado superficial vidriado y decoración. Los tipos comunes son vidriados en verde claro a amarillento, marrón verdoso y blanco amarillento, con decoración geométrica en negro. Las formas de vasijas son primordialmente ollas globulares, cuencos y platos con base plana. Una de las pocas descripciones de cerámica colonial tiene relación con Baeza de los Quijos (Porras 1974) y confirman la continuidad del contacto con el subandino durante la colonia. 


\section{PAtrón de ocupación PREhispánico en el SUbANDino}

El patrón de ocupación prehispánico en el borde oriental de la cordillera se encuentra asociado con los rasgos geográficos que presentan los diferentes medioambientes de los pisos ecológicos que tienen una específica y predominante variedad de especies vegetales. El medioambiente de páramo húmedo (3800 a $3200 \mathrm{msnm}$ ) entre la cuchilla de Cascacucho y la localidad de Papallacta, tiene como especie vegetal más abundante a la paja (Stipa ichu). No obstante, desde la creación de la reserva natural Cayambe-Coca la cubierta vegetal ha retomado parte de las características que posiblemente tuvo en el pasado. Las especies nativas como los árboles de queñua o quinoa (Polylepis rosaceae) han repoblado los microambientes de los espacios protegidos de los pequeños valles de origen glacial que están anexos a emanaciones de aguas termales. En este medioambiente se registraron muy pocas evidencias arqueológicas y al parecer solo fue utilizado como lugar de paso.

El piso por debajo del páramo, con clima templado del tipo bmh-M (bosque muy húmedo Montano), se extiende desde Papallacta hasta la hacienda de Chalpi a $2700 \mathrm{msnm}$. En el pasado la característica de este piso estaba asociado con el extenso cultivo de la papa para lo cual se utilizaron tradicionalmente las terrazas de origen prehispánico. De manera que el patrón ocupacional a lo largo del valle del río Papallacta tiene relación con la implementación de estrechas terrazas con la finalidad de suavizar la pendiente y poder edificar viviendas asociadas a campos de cultivo. La distribución de los sitios arqueológicos muestra una preferencia por espacios situados a cien metros por encima del lecho del río. Se advierte además un énfasis por sectores no comprometidos con quebradas que puedan producir deslaves.

Desde Chalpi, pasando por Baeza (1900 msnm) hasta la localidad del Reventador (1300 msnm) se extiende el tercer piso ecológico del tipo bmh-MB (bosque muy húmedo Montano Bajo) con vegetación de montaña y selva alta. En la apreciable variedad de árboles se destacan: Aliso (Alnus acuminata), cedro colorado (Cedrela odorata L.), cedro (Cedrela montana) choto (Citharexylum montanum), guato (Erythina edulis), morochillo (Guettarda tournefortiopsis), nogal (Juglans neotropica) y arbustos como la chilca (Baccharis macranta), menta (Mentha sp.), lengua de vaca (Bauhinia jenningsii) y guanto (Brugmansi arbórea). El paisaje está dominado por una disección arborescente de pequeños valles quebrados creado por cada uno de los tributarios de los ríos Papallacta-Quijos y Cosanga. Este tipo de drenaje dio lugar a la formación de pequeños espacios ondulados en las partes altas de las colinas y terrazas como las de Cuyuja y Baeza.

En los valles de los ríos Cosanga y Quijos la ocupación prehispánica tiene relación con la elaboración de terrazas mediante la construcción de muros de retención, dispuestas en forma paralela a los cursos de los ríos tributarios y principales. Los escasos espacios abiertos de terrazas naturales de apreciable extensión superficial fueron aprovechados para la erección de estructuras mayores como las presentes en el sitio de Oritoyacu y las colinas aisladas para una suerte de pucaras como en el sitio de Jambihuasi que se ubica en la confluencia de los ríos Papallacta y Quijos. Los sitios arqueológicos habitacionales en este tipo de medioambiente están situados en las terrazas naturales de mayor amplitud donde se construyeron muros de contención para conformar serie de terrazas artificiales con superficies horizontales. Con esta técnica se evitó el lavaje y movimiento de sedimentos en dirección a las corrientes fluviales. En los espacios con mayores pendientes las terrazas fueron utilizadas solo para cultivos.

En general los valles de las estribaciones orientales de la cordillera que corresponden a la ceja de montaña tienen pendientes abruptas y no existen espacios susceptibles de albergar extensas concentraciones habitacionales. A partir de los $2500 \mathrm{msnm}$ los pequeños valles subsidiaros del PapallactaQuijos y Cosanga, paulatinamente se ensanchan y sus laderas fueron ocupadas mediante la implementación de terrazas de mayor amplitud como el caso de las situadas en el área de Baeza.

A partir de la localidad El Reventador el valle del Quijos se abre completamente y el medioambiente predominante es de selva baja húmeda. El paisaje esta caracterizado por colinas bajas de sua- 
ves pendientes, intercaladas con corrientes de agua y pantanales, y terrazas altas a lo largo de los ríos principales. El patrón de ocupación prehispánico en esta región de la Amazonia baja, al cual no tuvo acceso la Fase Cosanga, se encuentra asociado a los espacios de tierra firme, es decir son culturas que ocuparon principalmente colinas y terrazas.

\section{CRONOlogía de LA EXPANSIÓN CosAnga}

Para la reconstrucción del periodo en el cual tuvo lugar la interacción de la fase Cosanga con la Sierra y la Amazonia baja, se debe analizar las fechas radiocarbónicas de los sitios asociados a material cultural Cosanga (Fig. 3). Para el efecto consideramos como probable lugar de origen de la fase Cosanga el valle del río Quijos. Por el momento al margen de esta fase no se tiene otra cultura identificada en la zona Norte del subandino.

Las fechas más antiguas provienen del sitio Borja Minda (Porras 1975) que se ubica a $1600 \mathrm{msnm}$ y en directa relación con el río Quijos. La ocupación del sitio se inicia a los 490 a.C. y continúa hasta el 1260 d.c. de manera que es muy probable que el área circundante a Borja sea el lugar de origen puesto que los conjuntos estructurales habitacionales y espacios de cultivos muestran una disposición arquitectónica planificada.

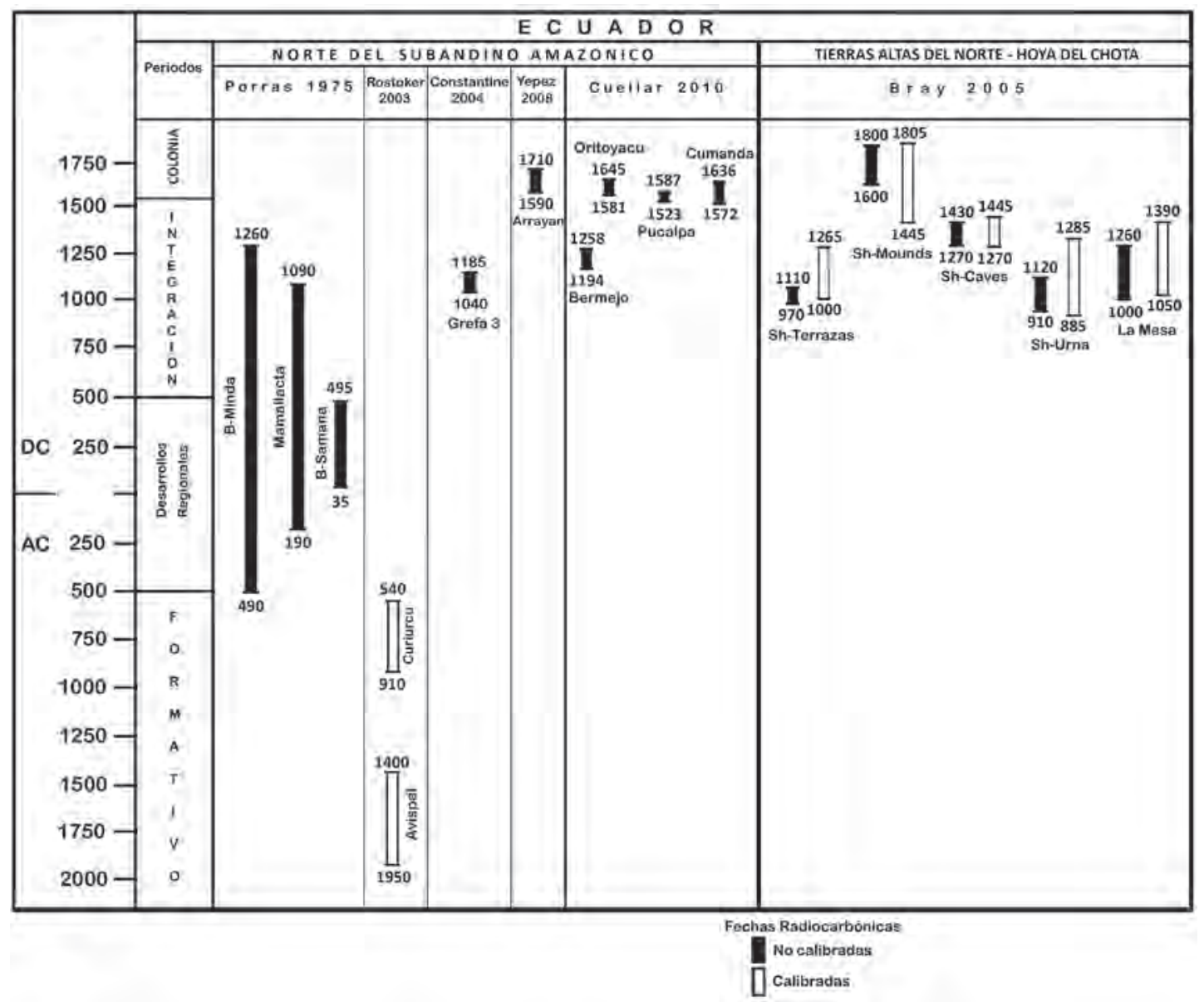

Figura 3. Cuadro cronológico mostrando el detalle de las fechas radiocarbónicas de sitios arqueológicos en la Hoya Interandina del Chota-Carchi y el subandino Norte del Ecuador. (Realizado por el autor). 
El siguiente sitio Mamallacta se encuentra en el área de Baeza, con un rango de ocupación entre el 190 a.C. al 1090 d.C. Posteriormente en la secuencia se ubica el sitio Banco Samana con una ocupación en el rango de 35-495 d.C. Con estas fechas se puede conjeturar que el inicio de la expansión o movimiento Cosanga aguas arriba en el valle de Quijos fue realizada a partir del 190 a.C.

Por su parte los sitios estudiados y descritos por Cuellar (2010) muestran que varios fueron utilizados durante la colonia. A través del sitio Bermejo fechado entre 1194-1258 d.C. (Cuellar 2010) se puede inferir que la completa ocupación en los valles Cosanga-Quijos y subsidiarios se consolidó alrededor de los 1194 d.C.

Mientrás el comercio o interacción con la sierra siguiendo el curso del río Papallacta hacia el paso de Guamani probablemente estuvo bastante arraigado a los 885 d.C. Las evidencias de cerámica Panzaleo o Cosanga recuperadas en el sitio multiétnico de Chapi situado en la hoya interandina del Chota (Bray 2005) y las obtenidas en los sitios asociados a la localidad del Inga en la hoya del Guayllabamba, muestran una expansión del comercio de productos del subandino.

Entre otras dataciones que pueden mencionarse con algunas reservas, son las obtenidas para los sitios Avispal y Curiurcu (Delgado 1999) que forma parte de las estribaciones subandinas. Estos sitios están situados al sur de la localidad de Cosanga, fuera de la sub-cuenca del río Quijos y asociados con la sub-cuenca del río Misahualli. El primero con material de la fase Cosanga tiene una fecha calibrada entre el 1950 a. C. y 1400 a. C., y el segundo entre el 910 a.C. al 540 a. C. (Rostoker 2005: 542). Al margen de estos sitios Yepez (2008: 214) tomando en cuenta los datos de Delgado (1999) menciona los sitios Arrayan con una datación de $1650 \pm 60$ d. C. y Pampas con $1870 \pm 85$ d. C., ambos con material Cosanga. Finalmente, de un contexto de Huila en las vecindades de Papallacta se indica una datación de 500 a.C. relacionada con Cosanga 1 (Almeida 2004).

\section{Contactos con la baja Amazonía}

La relación del subandino con las culturas del bajo Amazonas no es muy clara puesto que las evidencias coleccionadas en los trabajos de prospección marcan un límite muy definido de la dispersión de material Cosanga en el valle del río Quijos (Salazar et al. 1999). Este límite se encuentra prácticamente en las faldas del volcán Reventador en el área circundante a la confluencia del río Salado con el Quijos. La continuación de las prospecciones en la cordilla de Guacamayos, proporcionó evidencias que sugieren una ocupación aislada de grupos relacionados con Cosanga tipo Pituro-Bermejo. Los sitios registrados indican que desde el río Salado se trató de evitar los pisos inferiores del valle de Quijos y por necesidad de comunicación con los asentamientos aguas abajo, se utilizó las alturas de Guacamayos. Los fragmentos de cerámica se encuentran distribuidos desde los $1721 \mathrm{msnm}$ a partir de la localidad Sumaco Salahondo en dirección al área denominada Cooperativa Venceremos situada a 1150 msnm (Arellano 2000). El análisis tipológico de la cerámica permitió asociarlos al tipo Pituro-Bermejo del periodo tardío de la fase Cosanga. Aspecto que sugiere una interrupción en la comunicación entre río Salado y la localidad de El Reventador.

Los contactos de la sierra con la alta y baja Amazonía parecen haber sido frecuentes al inicio del período de Desarrollos Regionales. Evidencias de fragmentos de vasijas del tipo botella que se ubicaron en los niveles inferiores de las excavaciones en los sitios Pata-1 (Echeverria 1999), Yaguana (Carrera 2003), Aguarico (Arellano 2009) y San Antonio (Arellano y Tamayo 2004) situados en la region interfluvial entre el Aguarico y el Napo, sugieren que algunas fases culturales del Formativo Tardío de la hoya interandina del Guayllabamba tenían acceso a las tierras tropicales (Fig. 4). Los sedimentos acumulados en el área entre el río Salado y la localidad de San Francisco muestran evidencias de haber sido depositados mediante procesos de violenta remosión, característica que sugiere una disturbancia medioambiental en la cordillera, probablemente una combinación de sismos y eventos volcánicos relacionados con el volcán Reventador, con lo cual se interrumpió el contacto del subandino con la Amazonía. 


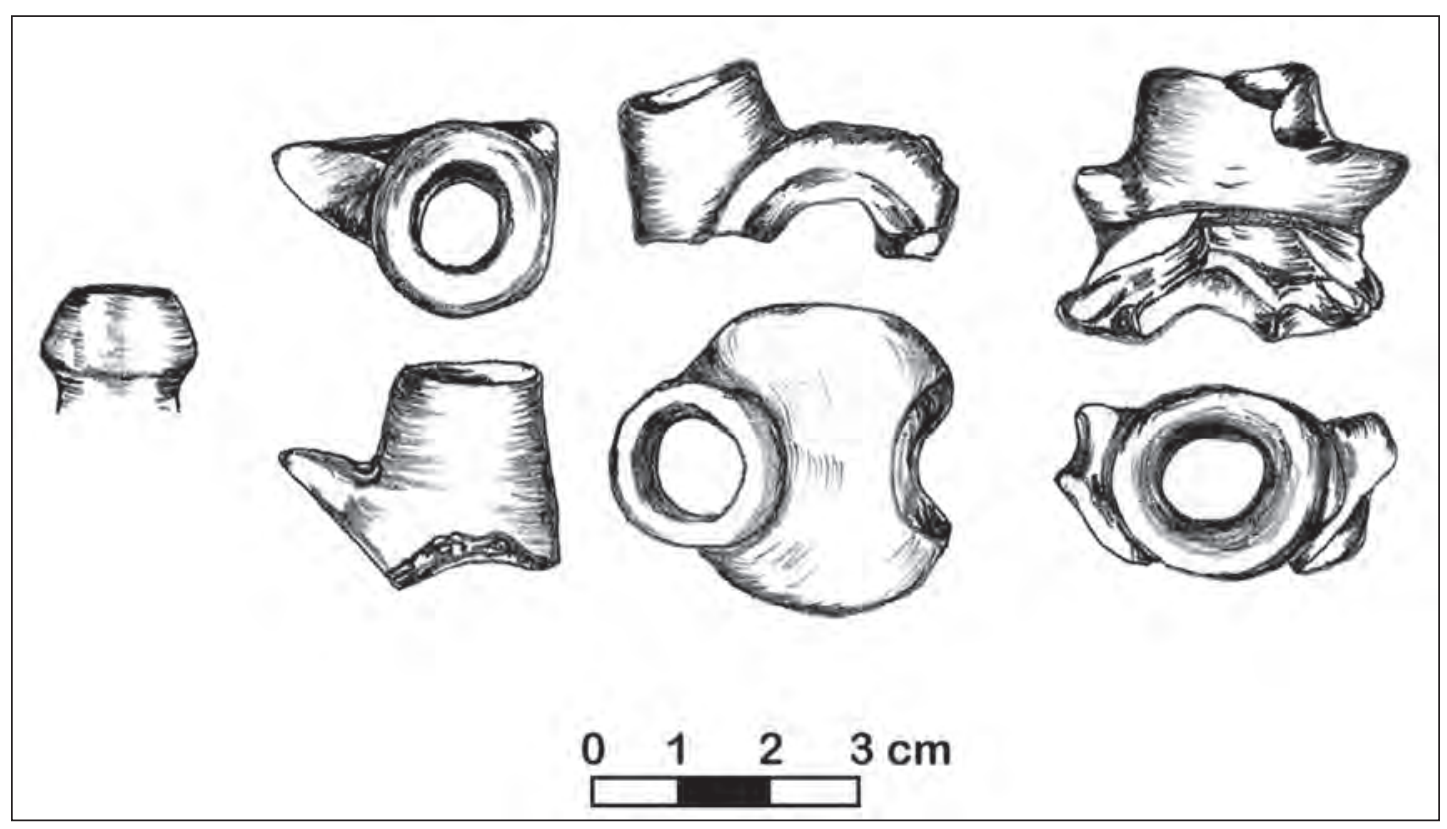

Figura 4. Fragmentos de picos de botella ubicados en los niveles inferiores de los sitios San Antonio asociados a la Cuenca del río Napo. (Modificado de Arellano \& Tamayo, 2004).

Al parecer un nuevo contacto se establece con la aparición de la tradición policroma en las áreas a lo largo del Napo y se vuelve a interrumpir durante la expansión de la tradición corrugada-falsocorrugada. No está claro si la tradición corrugada-falsocorrugada fue detenida por los Cosanga-Quijos u otra etnia, tal vez Cofanes, o por extremos eventos del Reventador. En el último caso los Cofanes parecen haber sido los intermediarios del subandino para la relación con la Hoya del Chota-Carchi hasta muy avanzada la colonia.

Las fechas radiocarbónicas de la baja Amazonía cercanos al borde oriental de la alta Amazonía y río Quijos son las que se obtuvieron para la fase Secoya en el sitio Luz de América ubicado en la sub-cuenca del río Shushufindi afluente del Aguarico; para el sitio Tank Farm situado en lago Agrio (Arellano 2003, 2008, 2009) y las mencionadas para la zona del proyecto Eno área situada entre los ríos Eno y Coca (Ochoa 2007).

La fase Secoya caracterizada por una cerámica de la tradición corrugada-falsocorrugada tiene un rango de 1390-1440 d.C. y el sitio Tank Farm que también pertenece a la misma fase tiene un rango de ocupación entre el 1230-1300 d. C. (Arellano, 2009). En relación con la tradición corrugada-falso corrugada Ochoa (2007) determinó cuatro periodos de ocupación en la zona del proyecto Eno: El primero del 250 al $640 \mathrm{AD}$, que vendría por el momento a corresponder a la primera ocupación de la tradición en la Amazonia baja del Norte. El segundo periodo 520 a 780 AD, el tercero 900 a 1410 y el cuarto entre el 1420 a 1650 AD.

Finalmente en las últimas estribaciones del piedemonte amazónico, en la orilla izquierda del Coca se ubica el sitio Pata 1 (Echeverría 1999) que está asociado a una tradición de cerámica con decoración punteada. La fecha calibrada más conservadora para el sitio tiene un rango entre el 1450 a.C. al 750 a.C. con un rango de probabilidad del 95\% (Rostoker 2003: 543), con la que estaría integrado al periodo del Formativo Tardío. También en las extensiones geográficas del piedemonte del Alto Napo, el sitio Grefa ubicado en el sector Yuralpa de la orilla derecha del río Napo que corresponde principalmente 
a un taller lítico con algunas evidencias de cerámica de bordes evertidos, tiene una fecha de 1040 a 1185 A.D. (Constantine 2004: 90).

\section{DiSCUSIÓN Y CONSIDERACIONES FINALES}

El origen de la fase Cosanga se encuentra bien establecida en el valle del Quijos, las fechas más antiguas provienen del sitio Borja Minda, a través del cual se infiere que primero se trataba de un grupo limitado caracterizado por una cerámica doméstica de paredes gruesas. Posteriormente se desarrolló la producción de la típica cerámica decorada de paredes delgadas. Los tipos Bermejo y Pituro descritos por Cuellar (2010), corresponden a tipos domésticos tardíos exclusivos de unidades familiares establecidas en el valle del Quijos-Cosanga. La difusión de la fase Cosanga fue realizada por medio de un movimiento migratorio y expansivo a lo largo y ancho del valle. El dominio de esta parte del subandino está registrado por la cantidad de sitios habitacionales.

A pesar de las diferencias tipológicas en los complejos cerámicos y variabilidad de los rangos cronológicos establecidos por los fechados para los sitios de la fase Cosanga, se observa una homogeneidad en la presencia porcentual mayoritaria y permanente de la cerámica típica diagnóstica de Cosanga. Aunque cada uno de los sitios en el subandino con material de la fase Cosanga presenta diferentes rangos cronológicos de ocupación, tienen una tendencia a complementarse unos con otros. Este aspecto posibilita la inferencia de las probables etapas de desarrollo cultural, expansión en el valle y posterior movimiento de esta particular cultura hacia las hoyas interandinas. De manera que es aceptable indicar que el foco inicial de origen y posterior difusión de esta variedad de cerámica es el valle del Quijos.

Según Porras (1975) la fase Cosanga utilizó probablemente para su desplazamiento hacia la Sierra ecuatoriana varios pasos de la cordillera oriental: A la Hoya interandina del Chota-Carchi, los pasos de Huaca - San Gabriel y Pimampiro. A la hoya interandina del Guayllabamba el paso de Guamaní, a Latacunga el paso de Chalupas. Finalmente hacia Ambato y Ríobamba el cañon del Pastaza. Sin embargo las únicas evidencias con material cultural Cosanga se encuentran hacia el paso de Guamani a través del río Papallacta. En esta ruta, el cruce del páramo húmedo que separa las vertientes occidental y oriental puede realizarse en una jornada de camino. Por otra parte, los contactos con la baja Amazonía parecen haber sido frecuentes en la dirección al Alto Napo por medio del río Hollín Grande y no hacia el Aguarico donde se encontraban obstáculos naturales como la cascada San Rafael y la inestabilidad del volcán Reventador.

Las evidencias en los espacios ocupados por las culturas amazónicas indican que durante el periodo de tiempo en el que la fase Cosanga se encontraba en su apogeo en el subandino, existió un movimiento de la tradición de cerámica corrugada-falsocorrugada desde la Amazonia Baja con dirección al piedemonte andino.

La ubicación de los sitios de la tradición corrugada-falsocorrugada sugiere una intención de movimiento hacia el subandino a través de la cuenca del río Quijos. Sin embargo, este desplazamiento fue detenido en el área circundante a la influencia destructiva del volcán El Reventador. En esta área no se encuentran evidencias de ocupaciones prehispánicas y los sitios que se encuentran fuera del radio de alcance de los materiales volcánicos, no tienen evidencias de la tradición corrugada-falsocorrugada. (Fig. 2).

Las fechas radiocarbónicas relacionadas con la tradición corrugada-falsocorrugada también permite inferir que su desplazamiento se inició entre el 880 a 970 d.C. y marcó sus límites territoriales en la Baja Amazonía alrededor del 1390 a 1440 d.C. Por lo menos en lo que respecta a la Amazonía norte, en el siglo XIV las culturas de esta tradición estuvieron establecidas en territorios definidos. El manejo del espacio amazónico estuvo concentrado más que todo al este del río Coca, en los microambientes de las diversas subcuencas subsidiarias de los ríos Aguarico y Napo. A partir de esta región interfluvial 
se generó un desplazamiento en dirección norte para cruzar el Aguarico y otro al sureste hacia la orilla izquierda del río Napo. La cerámica de la tradición corrugada-falsocorrugada tiene un rango de dispersión que alcanza hasta el borde oriental Norte de la alta Amazonia. Sin embargo, existen datos que podrían indicar un movimiento a partir de las cabeceras de la cuenca del Aguarico hacia la sierra cercana con la hoya del Chota a través de los sitios río Verde y La Bonita descritos por Delgado (2009: 111) y que los contactos inter-étnicos por los cuales se realizaron los primeros contactos con el bajo Amazonas fueron a través de esta ruta.

Los complejos culturales recuperados en los sitios de la tradición corrugada-falsocorrugada no tienen las evidencias para considerar una interacción temprana con las culturas del valle del Quijos. De la misma manera, los sitios Cosanga no tienen evidencias de materiales culturales de la Amazonía baja. Esta interacción se desarrolló en la época colonial por la presión europea en ambas direcciones. Desde el oeste española y del este portuguesa, y se consolidó tardíamente con la expansión colonizadora del siglo XVIII.

Otra zona de contacto se encuentra situado fuera de la vertiente del Cosanga-Quijos en el valle del río Misagualli, al cual se accede cruzando la cordillera de Guacamayos. Los sitios Curiurcu y Avispal con material formativo indican una temprana interacción con el piedemonte amazónico. En particular el sitio Avispal es importante por sus evidencias no solo de la fase Cosanga sino también de las fases Suno y Napo (Ivanhoe Energy Ecuador 2009: 293). Sin embargo, no se menciona la disposición estratigrafica del material cultural y la fecha radiocarbónica es controversial puesto que incluiría al sitio en el formativo temprano y la fase Suno pertenece al periodo de Desarrollos Regionales (Porras 1987b) y la fase Napo al periodo de Integración (Evans y Meggers 1968). Los sitios en el valle del río Misahualli pertenecen a la fase Suno que se desarrolló con características diferentes a la fase Cosanga. No tienen estructuras habitacionales similares a las de Cosanga del valle del río Quijos y su preferencia son las representaciones simbólicas en petroglifos (Porras 1985). Mientras en la orilla derecha del Napo, las evidencias obtenidas en el sitio Grefa (Constantine 2004), indican que los asentamientos estuvieron mayormente idéntificados con los pueblos amazónicos y en parte estuvieron especializados en la confección de instrumentos líticos.

El valle de Cosanga-Quijos está integrado a sitios que representan más que todo habitaciones permanentes con directa relación a superficies dedicadas a cultivos. La característica de la arquitectura doméstica Cosanga es la elaboración de terrazas para habitación e integración de cultivos. No existen evidencias arqueológicas de las especies cultivadas pero considerando las actuales especies nativas en cada uno de los pisos ecológicos de la zona (Fig. 5), se pueden inferir que algunas fueron empleadas como productos de intercambio. Si se toma en cuenta las especies domésticadas en cada piso ecológico al este de los Andes, se puede observar que las culturas del subandino no tuvieron una imperiosa necesidad de comunicarse con la baja Amazonía. Por el contrario, las culturas del bajo Amazonas necesitaban varios de los productos del subandino. De manera similar las culturas de las hoyas interandinas tenían necesidad de un intercambio con el subandino.

Las principales evidencias Cosanga (Panzaleo) en las hoyas de Guayllabamba y el Chota-Carchi, usualmente están representadas por vasijas muy bien elaboradas y de mediano tamaño. Esta característica podría tener relación con la especial atención que se dio a las actividades ceremoniales y funerarias (Bray 1995, 2005) y luego por la facilidad de transporte desde Quijos. Sin embargo a diferencia de los Andes centrales, en los Andes septentrionales no existen evidencias etnohistóricas de la presencia de etnias dedicadas a la exclusiva elaboración de vasijas de cerámica para comercialización. En este sentido, es probable que Cosanga tuvo una particular producción de cerámica para uso funerario, puesto que la mayor parte de los evidencias en la sierra están asociadas a tumbas.

La secuencia más antigua de la fase Cosanga en el sitio Borja Minda marca el punto de partida para la presencia Cosanga en el valle de Quijos. Se puede sugerir un movimiento a partir de los 190 a.C. hacia las partes altas de la cuenca siguiendo los diferentes cursos de los ríos Cosanga y 
Jorge Arellano / La interacción cultural prehispánica de los valles interandinos...

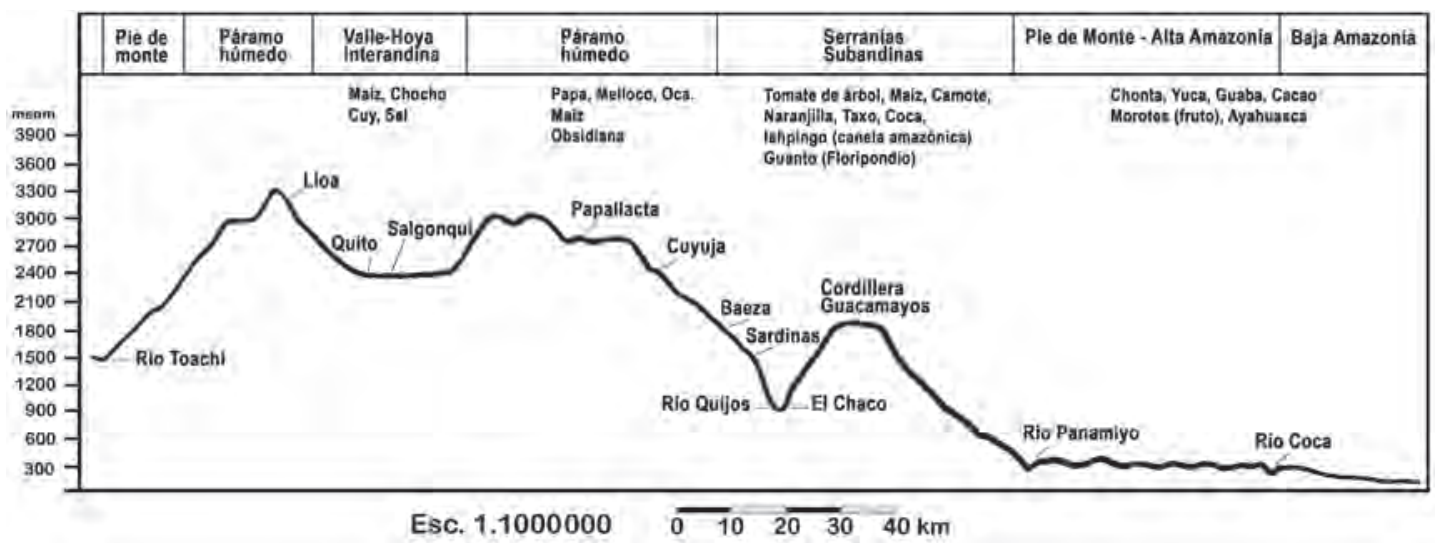

Figura 5. Perfil altitudinal Sierra-Amazonas, con los principales pisos ecológicos y productos agrícolas nativos y silvestres de consumo prehispánico. (Realizado por el autor).

Papallacta con el inicio de la ocupación de Mamallacta. Esto significa la temprana prioridad que tuvo esta fase para la interacción con los pueblos asentados en las hoyas interandinas. Hacia el 35 a.C. se produce un movimiento aguas abajo ocupando Banco Samana y partir de este período se extendió paulatinamente la ocupación en los valles subsidiarios con nuevos asentamientos. Estos asentamientos de Cosanga, que en realidad se los debe denominar Quijos, son ocupados hasta el período colonial.

El manejo del territorio y medioambiente Cosanga se puede determinar a través de la distribución de sus asentamientos en los valles de Papallacta, Cosanga y Quijos. El dominio de este territorio comprende desde los 3000 a 1500 msnm en las cercanías a la confluencia del río Azuela-Salado al río Quijos. Este límite aguas abajo del Quijos, se encuentra marcado por el área de influencia del alcance de los materiales expelidos por las erupciones del volcán El Reventador. Este volcán tiene un registro histórico de erupciones apróximadamente desde el año 1541 (Hall et al. 2004). Las últimas erupciones del volcán mostraron que los flujos de lava, deshechos, escombros y lodos de ceniza, utilizan canales de corrientes que se dirigen hacia el río Quijos (Fig. 6). De manera similar, la frecuencia de sismos que dan lugar a flujos de escombros y lodo están registrados en varios estudios relacionados con la destrucción del oleoducto trans-ecuatoriano. Por otra parte, estos desastres naturales son causantes de la remoción de taludes que tienen cubierta vegetal, además del represamiento de las aguas de los ríos Quijos y Due que originan inundaciones. Estos particulares fenómenos naturales tienen que haber sido en el pasado prehispánico los mayores obstáculos para asentamientos permanentes y para los contactos interétnicos entre el valle del Quijos y el piedemonte amazónico.

En conclusión se puede inferir por las fechas radiocarbónicas y material cultural de asentamientos prehispánicos en las hoyas interandinas, subandino y Amazonía, que existieron contactos entre los 800 a 550 a. C. Posteriormente existe un hiato hasta el 1000 d.C. y se vuelve a reiniciar este contacto con pueblos de la tradición polícroma que se encontraban en movimiento en dirección al Alto Napo. En ningún periodo el valle del río Quijos fue una ruta accessible para esta interacción. Las rutas probables fueron las cabeceras del río Cosanga atravesando la cordillera de Guacamayos hacia el Alto Napo, las nacientes del río Aguarico en conexión con la hoya interandina del Chota-Carchi, quizá esta última ruta haya sido más accessible y por la variedad de pucaras en la zona (Plaza 1977) sugestivamente con mayor control. Finalmente, es interesante observar que el registro de la ubicación de los asentamientos indígenas en el piedemonte amazónico al inicio y durante la colonia (Porras 1974: Lámina 6), muestra que evitaron el área circundante al volcán El Reventador y esta característica es sin lugar a dudas una tradición arraigada desde el Formativo Tardío. 


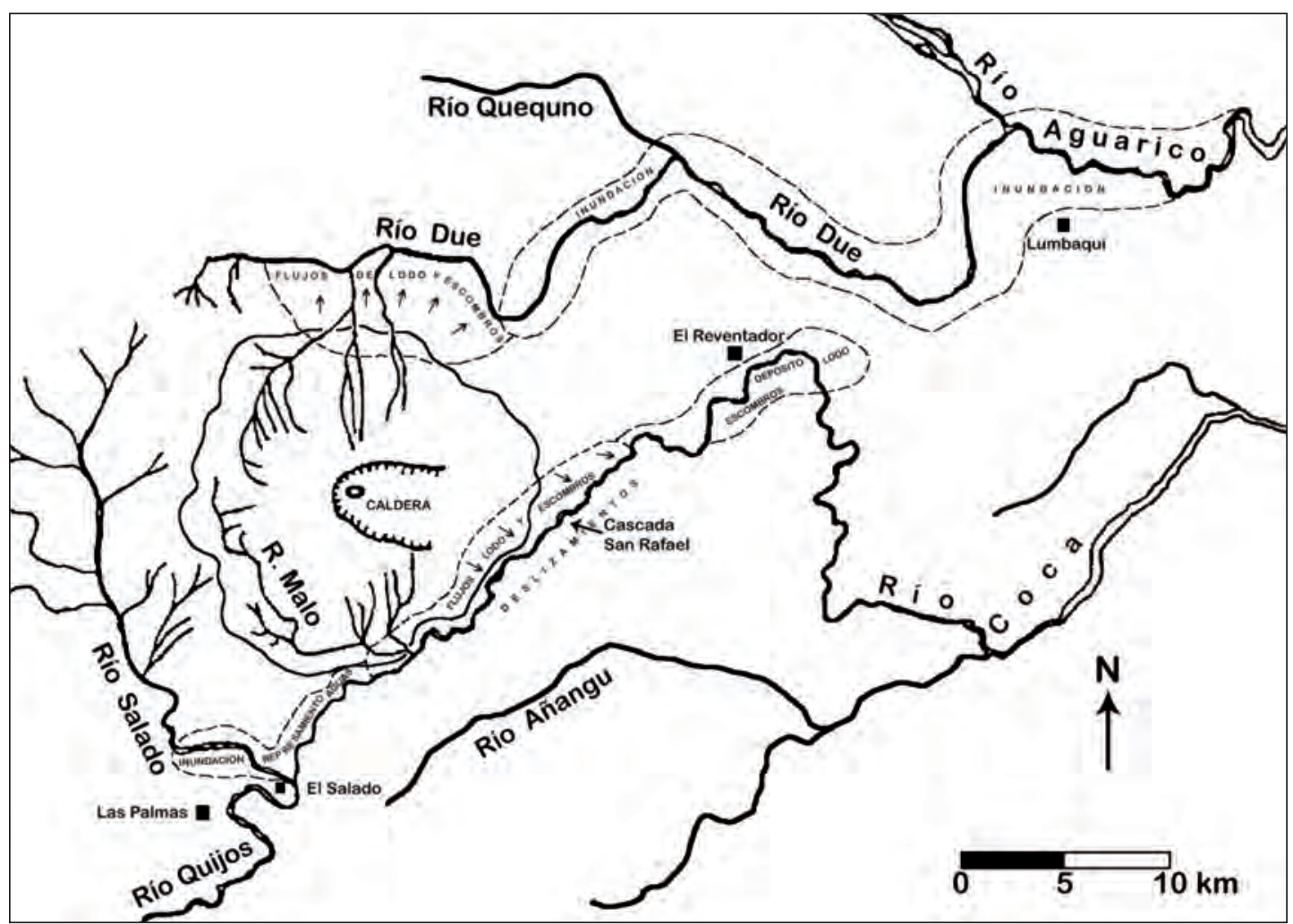

Figura 6. Mapa con detalle de los eventos medioambientales que se producen en el área de influencia del volcán El Reventador. (Realizado por el autor).

\section{Agradecimientos}

Deseo expresar mis agradecimientos al departamento de Antropología del Museo Nacional de Historia Natural de la Smithsonian Institution en la persona de la Dra. Mary Jo Arnoldi, Chair del Departamento, por darme la oportunidad de ser parte de la comunidad de investigadores de esta prestigiosa Institución. De la misma manera mis agradecimientos al Dr. Dennis Stanford, Dr. Paul Taylor y Dr. Ronald Bishop, por su apoyo para la conclusión del presente trabajo de investigación. Asi mismo mis agradecimientos al Dr. Mark Turber, Ing. Peter Ayarza y Lic. Cyana Zambrano de Walsh Environmental Scientists and Engineers Ecuador. Este trabajo de investigación está dedicado a la memoria de la Dra. Betty J. Meggers. 


\section{BibliogRAFIA}

ALMEIDA, Eduardo

2004 «Evidencias de vestigios arqueológicos monumentales de la fase Cosanga». Actas de de los Seminarios y Talleres científicos del 30 aniversario de ORSTOM/IRD en Ecuador. Quito.

ALMEIDA, Eduardo y Holger JARA

1984 El Pucara de Rumichuco. Miscelánea Antropológica Ecuatoriana. Serie Arqueológica 1. Quito: Banco Central del Ecuador.

ARELLANO, A. Jorge

1994 «Loma Pucara, a Formative site in the Cebadas Valley, Ecuador». National Geographic Research and Exploration 10 (1): 118-120.

1997 «Loma Pucara. Un asentamiento del Formativo Tardío en el valle de Cebadas, Sierra Central del Ecuador». Fronteras de Investigación, 1 (1): 78-100.

2000a Reconocimiento arqueológico en la alternativa Lumbaqui-Linares, de la línea del oleoducto de crudos pesados. Informe al Instituto Nacional de Patrimonio Cultural, Quito

2000b Reconocimiento arqueológico en Alto Pomasqui y Alto Yaruqui. Informe al Instituto Nacional de Patrimonio Cultural, Quito

2003 «Lago Agrio». En: Cuyabeno Ancestral: 123-164. Ediciones Simbioe-GAIA-WALSH, Quito.

2008 «Panorama de la Arqueología Amazónica del Ecuador, Primera Aproximación». Amazonia Peruana 15 (31): 101-121. Lima.

2009 Culturas Prehispánicas del Napo y el Aguarico, Amazonia Ecuatoriana. Lima: Ediciones Taraxacum Centro Cultural Pio Aza Lima - WALSH Ecuador.

ARELLANO, A. Jorge y Fernando TAMAYO

2004 Rescate y Monitoreo de la Línea de Flujo desde Yanaquincha Oeste hasta el CPF. Informe al Instituto Nacional de Patrimonio Cultural, Quito.

BRAY, Tamara L.

1995 «The Panzaleo Puzzle: Non-Local Pottery in Northern Highland Ecuador».Journal of Field Archaeology 22 (2): 137-156.

2005 «Multi-Ethnic Settlement and Interregional Exchange in Pimampiro, Ecuador». Journal of Field Archaeology 30 (2): 119-141.

CARRERA, Juan

2003 «Hacia el Suroeste de Nueva Loja». En: Cuyabeno Ancestral: 165-203. Ediciones Simbioe -GAIA WALSH. Ecuador.

CONSTANTINE C., Angelo

2004 La tecnología lítica del asentamiento prehistórico del sitio Grefa en la cuenca del río Canoayacu (Provincia de Napo, Cantón Tena, Parroquia Ahuano). Tesis de Grado; Guayaquil: Escuela Superior Politécnica del Litoral.

CUELLAR, Andrea M.

2006 The organization of agricultural production in the emergence of chiefdoms in the Quijos region, Eastern Andes of Ecuador, Dissertation: Pittsburgh: University of Pittsburgh.

2010 Pre-Hispanic Temporal Frameworks for the Analysis of Social Change in Ecuador and the Valle de Quijos. Comparative Archaeology Database: Pittsburgh: University of Pittsburgh.

DELGADO, Florencio

1999 Prospección sistemática, rescate y monitoreo arqueológico del Proyecto de Desarrollo Campo Villano, Bloque 10. Informe al Instituto Nacional de Patrimonio Cultural, Quito.

2009 «Arqueología, Ecuador: Cabeceras Cofanes-Chingual». Rapid Biological and Social Inventories. Report 21: 107 - 113. The Field Museum, Environmental Culture and Conservation. Chicago.

ECHEVERRÍA, José

1999 Informe Final del proyecto de Investigación Arqueológica realizada en la Plataforma Pata 1, y en el derecho de vía de la carretera y en la plataforma Palo Azul del Bloque 18 de Cayman International Company, Región Amazónica Ecuatoriana; Quito: Instituto Nacional de Patrimonio Cultural del Ecuador. 
EVANS, Clifford \& Betty J. MEGGERS

1968 Archaeological Investigations on the río Napo, Eastern Ecuador. Washington D.C.: Smithsonian Institution Press.

GUFFROY, Jean

1984 Investigaciones arqueológicas en el Sur de la provincia de Loja. Quito:

1987 Loja préhispanique: researches archéologiques dans les Andes méridionales de l'Equateur. Lima: Travaux de l'Institute Français d'Études Andines 32.

HALL, M., P. RAMÓN, P. MOTHES, J.L. LEPENNEC, A. GRACÍA, P. SAMANIEGO, P. y H. YEPES

2004 «Volcanic eruptions with little warning: the case of Volcan Reventador's Surprise November 3, 2002». Revista Geológica de Chile 31 (2): 349-358.

IVANHOE ENERGY ECUADOR

2009 Estudio de Impacto Ambiental y Plan de manejo Ambiental para perforación de Avanzada y Pruebas de Producción en el Bloque 20 de los Pozos IP-13,IP-15,IP-5A, IP-5B: Quito.

LEDERGERBER, Paulina

2006 «Ecuador Amazónico-Andino, Apropiación de paisajes y relaciones culturales». En: Gaspar Morcote-ríos, Santiago Mora \& Carlos Franky (eds.): Pueblos y Paisajes Antiguos de la Selva Amazónica, Universidad Nacional de Colombia - Taraxacum Washington D.C., Bogota.

OCHOA, Myriam

2007 «Redefiniendo la Fase Pastaza». II Congreso de Antropología y Arqueología. Balance de la última década, aportes, retos y nuevos temas 1: 463-495; Quito: ABYA Yala - Banco Mundial Ecuador.

ONTANEDA LUCIANO, Santiago

2002 El cacicazgo Panzaleo como parte del área circumquiteña. Quito: Ediciones del Banco Central del Ecuador.

PLAZA SHULLER, Fernando

1976 La incursión Inca en el Septentrión Andino Ecuatoriano, Antecedentes arqueológicos de la convulsiva situación del contacto cultural. Otavalo: Serie Arqueológica. Instituto Otavaleño de Antropología.

PORRAS, Pedro I.

1974 Historia y Arqueología de la Ciudad Española Baeza de los Quijos. Quito: Imprenta Lexigrama.

1975 Fase Cosanga. Quito: Pontificia Universidad Católica del Ecuador.

1985 Arte Rupestre del Alto Napo, Valle de Misahuallí. Quito: Artes Graficas Señal.

1987a Investigaciones Arqueológicas a las Faldas del Sangay, Tradición Upano. Quito: Artes Gráficas Señal.

1987b Nuestro Ayer, Manual de Arqueología Ecuatoriana. Quito: Artes Gráficas Señal.

Rostain, Stephen

1999 «Secuencia arqueológica en montículos del valle de Upano en la Amazonía Ecuatoriana». Bulletin de l'Institut Francais d'Etudes Andines IFEA, 28 (1): 53 - 89.

ROSTOKER, Arthur

2003 «Formative Period Chronology for Eastern Ecuador». En: Jeffrey Quilter (ed.): Archaeology of Formative Ecuador. Dumbarton Oaks Research Library and Collection. Washington D.C.

SALAZAR, ERNESTO, A.J. ARELLANO, M. OCHOA y O. MANOSALVAS

1999 Informe del Reconocimiento Arqueológico de la Línea del Oleoducto Ecuatoriano y Zonas Adyacentes. Instituto Nacional de Patrimonio Cultural. Quito.

SALAZAR, Ernesto

1998 «De vuelta al Sangay, Investigaciones arqueológicas en el Alto Upano, Amazonia Ecuatoriana». Bulletin de l'Institut Francais d'Etudes Andines IFEA, 27 (2): 213 - 240.

2008 «Pre-Columbian Mound Complexes in the Upano River Valley, Lowland Ecuador». En: Helaine Silverman \& William H. Isbell (eds.): Handbook of South American Archaeology. Springer. New York

YEPEZ, Alden

2008 Wandel und Kontimität der Keramic ans den Flusstälern Cosanga und Quijos, Provinz Napo, Ecuador. These. Bonn: Universität zu Bonn. 